\title{
Anti-Stokes Excitation of Solid-State Quantum Emitters for Nanoscale Thermometry
}

\author{
Toan Trong Tran, ${ }^{1, *}$ Blake Regan, ${ }^{1}$ Evgeny A. Ekimov, ${ }^{2}$ Zhao Mu, ${ }^{3}$ Zhou Yu, ${ }^{3}$ Weibo Gao,${ }^{3}$ Prineha Narang, ${ }^{4}$ \\ Alexander S. Solntsev, ${ }^{1}$ Milos Toth, ${ }^{1}$ Igor Aharonovich ${ }^{1}$ and Carlo Bradac ${ }^{1}$ \\ 1 School of Mathematical and Physical Sciences, University of Technology Sydney, Ultimo, NSW, 2007, Australia \\ 2 Physics, RAS Kaluzhskoe Road 14, Troitsk, 142190, Russia \\ 3 Division of Physics and Applied Physics, School of Physical and Mathematical Sciences, Nanyang Technological University, 637371, \\ Singapore \\ 4 John A. Paulson School of Engineering and Applied Sciences, Harvard University, Cambridge, MA, USA \\ Author e-mail address: trongtoan.tran@uts.edu.au \\ Author address: UTS Building 4, 745 Harris Street, Broadway, Ultimo NSW 2007, Australia \\ Author phone number: +61424357361
}

Abstract: We report the first demonstration of Anti-Stokes excitation on a single solid-state quantum emitter-namely the germanium-vacancy center in diamond and its application as a highsensitive nanoscale thermal sensor. ( $) 2019$ The Author(s)

OCIS codes: (270.0270) Quantum Optics, (300.6250) Spectroscopy: Condensed Matter, (160.2220) Materials: Defectcenter materials

\section{Full Abstract}

Stokes and Anti-Stokes emission are fundamental phenomena widely used to study the physico-chemical and optical properties of materials. Stokes (Anti-Stokes) photoluminescence (PL) occurs when the energy of the emitted photons is lower (higher) than that of the absorbed ones [1]. In the Anti-Stokes case, the extra energy that causes upconversion of the photons can be acquired through a variety of mechanisms, ranging from multi-photon absorption to Auger recombination and phonon absorption. The latter, relevant to this work, is illustrated in Figure 1A, B. A photon with energy $h v_{\text {exc }}$ at the long-wavelength tail of the absorption spectrum excites an electron from a thermally-populated first vibronic state $\left(n_{0}=1\right)$ of the electronic ground state $E_{0}$, to the bottom manifold $\left(n_{1}=0\right)$ of an excited electronic state $E_{1}$ [red arrow]. The system then returns to the ground state via spontaneous emission of an up-converted photon with a mean energy $h v_{s e}>h v_{\text {exc }}$ [yellow arrow]. This phonon-assisted Anti-Stokes excitation process scales exponentially with temperature and is the bedrock of a variety of fundamental studies (e.g. cavity quantum electrodynamics), as well as practical applications such as optical cryocooling, bioimaging and Raman spectroscopy. However, Anti-Stokes photoluminescence (PL) is inherently inefficient, and all work done to date on solid-state defects has been focused on ensembles [2-4] rather than individual point defects.

Here, we demonstrate that Anti-Stokes PL can be used to study isolated quantum systems - specifically atomlike color centers in diamond, over a large range of temperatures [5]. We explore the mechanism for some of the most studied diamond defects, the nitrogen-vacancy (NV) the silicon-vacancy (SiV) and the germanium-vacancy $(\mathrm{GeV})$ center. We show that Anti-Stokes excitation of selected diamond color center is an efficient process, detectable by standard photoluminescence spectroscopy and leverage this finding to demonstrate upconversion PL from a single, isolated $\mathrm{GeV}$ defect. We show that the Anti-Stokes excitation process is thermally-activated and proceeds through a phonon-photon absorption pathway rather than through multi-photon absorption. We exploit the high Anti-Stokes excitation efficiency to introduce an innovative approach for all-optical nanoscale thermometry based on the temperature-dependence of the Anti-Stokes to Stokes PL intensity ratio. Our technique outperforms all other previously reported all-optical nanothermometry methods. 
A

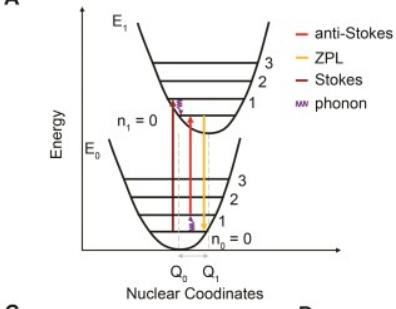

C

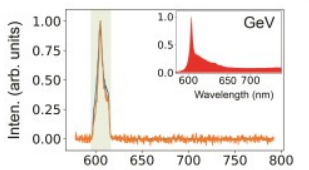

D
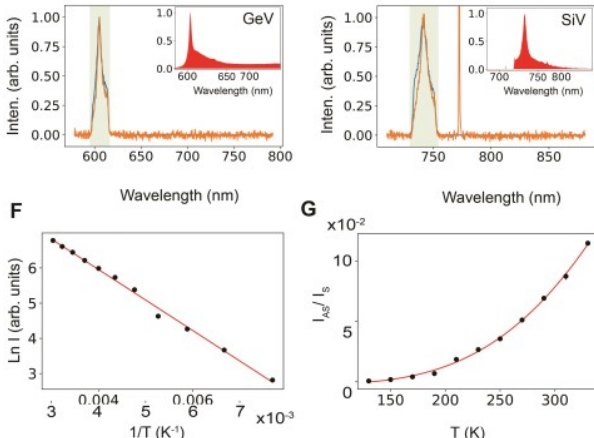

$$
\text { G }
$$

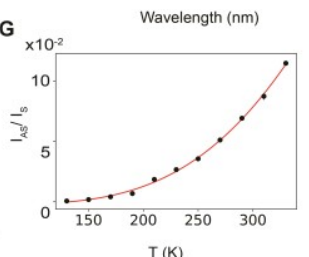

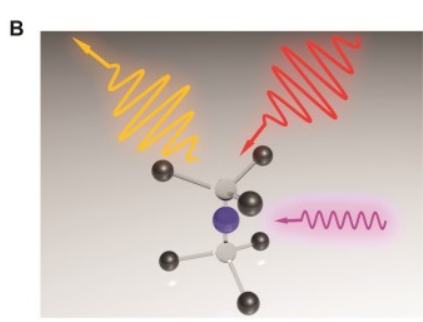

E
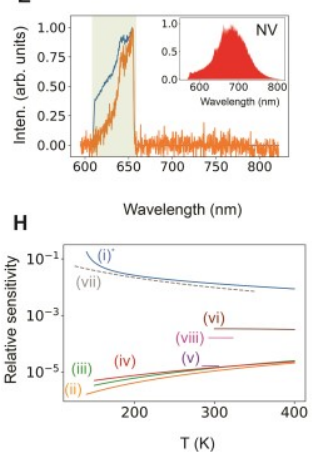

Fig. 1 Stokes and Anti-Stokes luminescence processes for color centers in diamond. (A) Energy diagram of representative electronic and vibrational energy levels for a diamond color center. The arrows show the lower (higher) energy of the Stokes (Anti-Stokes) photons with respect to the ZPL energy. In the Anti-Stokes case, the additional energy is acquired via phonon(s) absorption. (B) artistic representation of the AntiStokes mechanism for a diamond color center which absorbs a lower-energy photon [wavy line, red] and emits a higher-energy one [wavy line, ocher] upon absorption of a phonon [wavy line, purple]. (C-E) Photoluminescence spectra of the ZPL for nanodiamond GeV (C), SiV (D) and NV (E) centers under Stokes [blue] and Anti-Stokes [ocher] excitation (the full PL spectrum under Stokes excitation is shown in the relative inset). The ZPLs ( $605 \mathrm{~nm}$ for $\mathrm{GeV}, 739 \mathrm{~nm}$ for $\mathrm{SiV}$ and $639 \mathrm{~nm}$ for NV) are spectrally filtered by means of bandpass filters (semitransparent rectangular boxes). Characterization of the Anti-Stokes GeV-based nanothermometer. (F) Temperature dependence of the PL intensity signal upon Anti-Stokes excitation (637-nm wavelength). The PL intensity was measured by monitoring the GeV's ZPL (605 nm) isolated with a bandpass filter. The data fit well the Arrhenius-type equation $A e^{-\left(E_{a} / k_{B} T\right)}$, where the activation energy $E_{a}=102.96 \mathrm{meV}$ is fixed to coincide with the difference in energy between the excitation laser and the germanium-vacancy's ZPL. (G) Plot of the Anti-Stokes to Stokes PL ratio as a function of temperature. The ratio fits an exponential curve: $a+b e^{-\left[c /\left(T-T_{0}\right)\right]}$, granting the method an extremely high sensitivity. The error bars of plots in $(\mathrm{F})$ and $(\mathrm{G})$ are represented as vertical, blue bars, and are mostly equivalent to or smaller than the size of the data points. (H) Relative sensitivity plotted vs temperature for several different systems: our $I_{A S} /\left.I_{S}\right|_{G \theta V}$ measurement $(i)^{*}$, the frequency of the GeV ZPL in our Stokes PL spectra (ii), and the equivalent measurement from the literature (iii), the ZPL wavelength shift of the $\mathrm{SnV}$ (iv) and of the $\mathrm{SiV}$ center (v), the intensity of the NV ZPL (vi), the Raman $I_{A S} / I_{S}$ ratio achieved for a bulk thermometer (vii) and the spectral shift of quantum dots (viii). The literature data are plotted over the entire temperature range demonstrated in each paper.

\section{Summary}

In conclusion, we present the first demonstration of Anti-Stokes excitation on a single solid-state quantum emitternamely the germanium-vacancy center in diamond and its application as a high-sensitive nanoscale thermal sensor.

\section{Acknowledgements}

Financial support from the Australian Research council (via DP180100077, DE180100810 and DE180100070), the Asian Office of Aerospace Research and Development grant FA2386-17-1-4064, the Office of Naval Research Global under grant number N62909-18-1-2025 are gratefully acknowledged. E. A. Ekimov is grateful to RFBR for the financial support under Grant No. 17-52-50075. The authors thank Marcus W. Doherty, Neil B. Manson and Jeff Reimers for useful discussions.

\section{References}

1. G. G. Stokes, "On the Change of Refrangibility of Light," Philosophical Transactions of the Royal Society of London 142, 463-562 (1852).

2. Y. Xu, H. Naramoto, K. Narumi, K. Miyashita, T. Kamiya, and T. Sakai, "Strong anti-Stokes luminescence from H+-irradiated diamond," Appl. Phys. Lett. 83, 1968-1970 (2003).

3. Q. Wang, Q. Zhang, X. Zhao, X. Luo, C. P. Y. Wong, J. Wang, D. Wan, T. Venkatesan, S. J. Pennycook, K. P. Loh, G. Eda, and A. T. S. Wee, "Photoluminescence Upconversion by Defects in Hexagonal Boron Nitride," Nano Lett. (2018).

4. $\quad$ D. V. Seletskiy, S. D. Melgaard, S. Bigotta, A. Di Lieto, M. Tonelli, and M. Sheik-Bahae, "Laser cooling of solids to cryogenic temperatures," Nat. Photon. 4, 161 (2010).

5. T. T. Tran, B. Regan, E. A. Ekimov, Z. Mu, Z. Yu, W. Gao, P. Narang, A. S. Solntsev, M. Toth, and I. Aharonovich, "Anti-Stokes excitation of solid-state quantum emitters for nanoscale thermometry," arXiv preprint arXiv:1810.05265 (2018). 